\title{
Consideraciones sobre algunas metodologias habituales para el análisis de tablas de movilidad social. Ventajas $y$ limitaciones de una alternativa basada en cadenas de Markov ${ }^{1}$
}

\begin{abstract}
Considerations on some methodologies usually used for the study of social mobility tables. Benefits of an alternative based on Markov's Chains
\end{abstract}

\author{
GERMÁN ROSATI \\ Universidad Nacional de General Sarmiento (UNGS) \\ gfrosati@yahoo.com.ar (ARGENTINA)
}

Recibido: 27.02 .2010

Aceptado: 12.01.2011

\section{RESUMEN}

El problema de la «movilidad social» constituye un tópico clásico en la ciencia social y ha tenido diversos abordajes. En este trabajo se presentan algunas herramientas metodológicas para el análisis de la movilidad social en matrices de transición.

Se discutirán los alcances y limitaciones de algunas metodologías clásicas de análisis: análisis de «entradas» y «salidas; el índice de movilidad/ estabilidad social; el índice basado en el coeficiente Q de Joule desarrollado por Andrea Tyree y los modelos log-lineales. Estas herramientas funcionan bajo el supuesto de «independencia estadística» entre los momentos de la matriz de transición. Se intentará mostrar la utilidad que las cadenas de Markov poseen, en tanto permiten «flexibilizar» (al menos en teoría) estos supuestos, al trabajar sobre distribuciones probabilísticas de movilidad (llamadas matrices de transición). Por ello se propondrán algunos elementos para la construcción de un índice basado en estas distribuciones probabilísticas y se compararán sus resultados con las metodolo-

\footnotetext{
${ }^{1}$ Agradezco los comentarios y sugerencias a este trabajo de los dos evaluadores anónimos.
} 
gías anteriores. Se ilustrarán algunos de los resultados con datos secundarios provenientes de otros trabajos de investigación.

\title{
PALABRAS CLAVE
}

Movilidad social - cadenas de markov - Estructura social - Independencia estadística

\begin{abstract}
Social mobility is a classic issue in social science and it have had different approaches. This paper presents some methodological tools for the analysis of social mobility based on transition matrixes.

We will try to discuss the limits and scopes of some classical methodologies: inflows and outflows analysis, the mobility/ stability index, the one based on Joule's Q coefficient wich has been developed by Andrea Tyree and log-linear models. These methodologies work under the assumption of «statistical independence » between the different moments of the mobility matrixes. We will try to show the utility that Markov`s chains have, because they allow to flexibilize these assumptions working with probabilistics mobility distribution (called «mobility matrixes»). We will propose some elements towards the construction of a «mobility index» and we will compare the results with the others methodologies of analysis. We will illustrate this ideas with secondary data wich come from other works.
\end{abstract}

\section{KEY WORDS}

Social mobilty - Markov chains - Social structure- Statistical independence

\section{INTRODUCCIÓN}

El problema de la «movilidad social» es un tema clásico de la ciencia social. Las controversias y discusiones a su alrededor no solamente provienen de la contraposición ente diferentes marcos teóricos sino también en torno a problemas «operacionales», es decir, a problemas relacionados con su medición y su contratación empírica.

En efecto, las discusiones alrededor de la construcción de medidas que puedan incorporar la totalidad (o al menos, la parte más sustantiva) de sus determinaciones son tanto o más extensas que las discusiones teóricas. 
En este trabajo nos centraremos en la dimensión «metodológico- técnica» de la movilidad social dejando de lado las discusiones teóricas al respecto ${ }^{2}$. Tampoco centraremos el siguiente trabajo en las discusiones sobre la magnitud, características, formas y manifestaciones de la movilidad social en Argentina. Nos ocuparemos, en cambio, de algunas de las discusiones que se han dado en los estudios sociales argentinos y latinoamericanos, acerca de las formas de «cuantificar», «interpretar» y «analizar» los procesos vinculados a la movilidad social a partir de matrices de movilidad.

Proponemos, entonces, un breve recorrido por cuatro de los métodos «clásicos» ${ }^{3}$ para el análisis de la movilidad social: a) los análisis de «inflows» y «outflows»; b) el índice de (in)movilidad social; c) el índice basado en $Q$ de Jou$l e$, propuesto por Andrea Tyree y d) los modelos de análisis log-lineal.

El objetivo, entonces, es lograr una reflexión sobre algunos de los supuestos estadísticos y conceptuales que están por detrás de estos métodos. Expondremos algunas de sus limitaciones e intentaremos aportar algunos elementos que permitan evaluar las potenciales ventajas del intento de modelización de la movilidad social a partir de la utilización de los procesos de Markov.

El trabajo constituye una primera aproximación al problema y, como tal, tiene más interrogantes que certezas. Intentará funcionar como disparador de algunas ideas y exponer algunas potencialidades que consideramos relevantes de las cadenas de Markov para abordar este tema clásico de la ciencia social.

En ese sentido es necesario observar que el uso de estos modelos no es «novedoso» en el análisis de la movilidad social. Sin embargo, resulta interesante comprobar que en América Latina las cadenas de Markov no parecen haber sido totalmente aprovechadas.

Si tomamos el ejemplo de la Argentina, puede notarse que en los estudios «clásicos», los más importantes y los más recientes, sobre la temática (Germani 1961; Beccaria 1978; Kessler y Espinoza 2007; Jorrat 1995 y 2008, Golovanevsky 2008) el instrumental de análisis se circunscribe a los cuatro métodos que hemos citado.

Por otro lado, consideramos que en algunos de los estudios en los que el uso de las cadenas de Markov está presente (por ejemplo, Baltazar, Grillo Astudillo y Malaver 2003), no parece haberse avanzado en una adecuada interpretación de sus ventajas y/ alcances metodológicos (y conceptuales) en relación a los restantes métodos de análisis. Asimismo, tampoco se ha producido a nuestro juicio

\footnotetext{
${ }^{2}$ Esto quiere decir que no nos centraremos en las discusiones que se han generado sobre los sistemas clasificatorios (neoweberianos, neomarxistas, etc.) de las posiciones de «clase». Tampoco entraremos a discutir un tema, sumamente importante, relacionado con el anterior: la tendencia latente de buena parte de los estudios de movilidad social a considerar el sistema de las posiciones de clases como un «continuum» susceptible de ser «ordenado» y no como sistemas de relaciones sociales cualitativamente diferentes. Una discusión al respecto puede encontrarse en Echeverría Zabalza (1999).

${ }^{3}$ Dejamos fuera del análisis aquellos modelos basados en diversos tipos de regresiones (lineales, logísticas, etc.) dado utilizan supuestos y estimadores que difieren de aquellos analizados aquí. Al mismo tiempo, la lógica de interpretación de sus resultados también resulta diferente.
} 
una reflexión lo suficientemente adecuada acerca de los diferentes supuestos conceptuales en relación a la movilidad social que se encuentran por detrás de los métodos de análisis «tradicionales» de las tablas de movilidad.

En efecto, ¿qué supone plantear una hipótesis nula de «independencia estadística» entre orígenes y destinos?, ¿que supuesto conceptual se encuentra detrás del intento de «controlar» el efecto de la diagonal principal, las distribuciones rinconales, etc., a través de modelos de tipo log-lineal? Y al mismo tiempo, ¿cuáles son las implicancias conceptuales de intentar construir una matriz de transición a partir del marco que brindan las cadenas de Markov? Es en estas dimensiones, especialmente, en que esperamos centrar nuestra reflexión.

\section{PLANTEO DEL PROBLEMA}

Suele definirse a la «movilidad social» de formas diversas, pero generalmente se lo hace de manera descriptiva. Podemos encontrar «movilidad social educativa», «movilidad social ocupacional», «movilidad de ingresos» y demás movilidades por el estilo. Si bien uno de los supuestos metodológicos y operacionales generalmente aceptados en torno a la movilidad social (ocupacional) es que se trata, en general, de movilidad «intergeneracional» (es decir, los cambios de posición entre generaciones de sujetos $)^{4}$, también puede definirse a la «movilidad» como los sucesivos cambios de estado o de posición que un mismo sujeto experimenta a lo largo de un período $t$. Las consideraciones que presentamos deberían ser pasibles de ser aplicadas a ambos «tipos» de movilidad.

Uno de los insumos básicos para su abordaje son las tablas o matrices de movilidad: tablas de contingencia (generalmente) bivariadas, donde una de las variables (la independiente) representa la posición o estado del sujeto en un momento de tiempo anterior ( $\mathrm{t} 0$ ), y la variable dependiente, representa la posición o estado en un momento de tiempo posterior ( $\mathrm{t} 1)$.

El problema al enfrentarse a este tipo de tablas es la dificultad de encontrar formas de interpretar correctamente la información que brindan. Jorrat (2001), distingue tres grandes generaciones en los análisis sobre movilidad social. Si bien el autor desarrolla algunos de los hallazgos y conclusiones de estas generaciones, nosotros nos centraremos en las herramientas metodológicas utilizadas.

La primera basó sus estudios sobre el estudio de tablas de contingencia, generalmente construidas sobre clasificaciones tripartitas. La metodología generalmente utilizada se centró en el análisis de los porcentajes de «entradas» y «salidas». Posteriormente se incorporaron exploraciones sobre las «chances» de movilidad intentando controlar las diferencias en los marginales de las tablas, elaborando índices de (in)movilidad social.

\footnotetext{
${ }^{4}$ Una de las formas de operacionalizar el concepto de movilidad social a través de la investigación por encuestas es preguntar al entrevistado acerca de la ocupación de su padre, alrededor de una edad determinada (generalmente durante la adolescencia) (Cfr. Jorrat 2005).
}

EMPIRIA. Revista de Metodología de Ciencias Sociales. N. ${ }^{\circ}$ 22, julio-diciembre, 2011, pp. 67-90. ISSN: 1139-5737 
La segunda generación incorpora como técnicas de análisis el uso de datos «continuos» de panel y, especialmente, el estudio de modelos de «path».

Una tercera generación se caracterizó, hacia los años '70, por la utilización de grandes muestras nacionales, permitiendo las comparaciones internacionales. La innovación metodológica de esta generación estuvo constituida por la utilización de modelos log-lineales, con los que intentaban controlar y detectar efectos de «herencia», jerárquicos, sectoriales y de afinidad. Como puede verse, el instrumental metodológico que consideraremos en este trabajo es producto de varias generaciones en el análisis de la movilidad social.

Para ejemplificar algunas de las consideraciones aquí realizadas utilizaremos como fuente de datos las tablas presentes en un estudio reciente (Jorrat 2008) acerca de la movilidad social en Argentina durante los años 2003 y 2004. Sobre el final, incorporaremos los resultados de un estudio más lejano en el tiempo (Jorrat 2001). El objetivo será lograr la comparación de algunos de nuestros resultados con los obtenidos en ambos estudios.

\section{ANÁLISIS DE «OUTFLOWS-INFLOWS»}

Los análisis de «entradas» y «salidas» constituyen la herramienta más simple al momento de analizar una tabla de movilidad. En efecto, brindan un primer panorama de algunas de las tendencias subyacentes en la misma. De esta manera, suele constituir el primer acercamiento del investigador con la información «bruta».

Uno de los análisis pioneros en Argentina acerca de la temática de la movilidad social (Germani 1965) hace uso extensivo de estos indicadores y les atribuye un carácter puramente descriptivo, si bien reconoce la necesidad de incorporar indicadores de carácter explicativo. En efecto, Germani introduce la posibilidad de utilizar el índice de inmovilidad basado en el supuesto de independencia estadística5. De igual manera, Beccaria (1978) se concentra en el análisis de las entradas y salidas de las tablas de movilidad. Asimismo encara, la utilización de algunos índices para controlar ciertos efectos y distorsiones en las tablas de movilidad.

La distribución siguiente representa los cambios en la estructura social (orígenes, es decir, tipo de ocupación del padre, y destinos, o sea, tipo de ocupación del hijo) de una muestra representativa de la población (masculina y femenina) entre 24 y 69 años en el total de la República Argentina. Surge de dos encuestas llevadas adelante en 2003 y 2004 por el Centro de Estudios de Opinión Pública (CEDOP) del Instituto Gino Germani de la Universidad de Buenos Aires.

${ }^{5}$ «A este tipo de análisis [se refiere al análisis de porcentajes de entradas y salidas] se oponen otros que se fundan sobre determinados supuestos, por ejemplo el de movilidad perfecta: en este caso los índices no miden las proporciones que estrictamente pasan de un nivel a otro, sino que comparan la movilidad empírica con aquella que se daría en el caso que las posiciones (...) se distribuyeran al azar...»(Germani 1961: 337).

EMPIRIA. Revista de Metodología de Ciencias Sociales. N. ${ }^{\circ} 22$, julio-diciembre, 2011, pp. 67-90. ISSN: $1139-5737$ 
Cuadro 1. Movilidad desde la ocupación o clase de los padres hacia la de los encuestados. Ambos sexos (absolutos). Argentina, 2003-04

\begin{tabular}{|l|r|r|r|r|r|r|}
\hline \multirow{2}{*}{\multicolumn{1}{|c|}{ Destino }} & \multicolumn{7}{|c|}{ Origen } \\
\cline { 2 - 8 } & $\begin{array}{c}\text { Clase de } \\
\text { Servicios }\end{array}$ & $\begin{array}{c}\text { No } \\
\text { Manuales }\end{array}$ & $\begin{array}{c}\text { Pequeña } \\
\text { burguesía }\end{array}$ & $\begin{array}{c}\text { Manuales } \\
\text { calificados }\end{array}$ & $\begin{array}{c}\text { Manuales } \\
\text { no } \\
\text { calificados }\end{array}$ & Total \\
\hline Clase de Servicios & 72 & 32 & 62 & 38 & 27 & 231 \\
\hline No Manuales & 56 & 62 & 95 & 112 & 56 & 381 \\
\hline Pequeña burguesía & 38 & 37 & 185 & 71 & 85 & 416 \\
\hline Manuales calificados & 18 & 22 & 72 & 99 & 65 & 276 \\
\hline Manuales no calificados & 8 & 11 & 83 & 84 & 187 & 373 \\
\hline Total & 192 & 164 & 497 & 404 & 420 & 1.677 \\
\hline
\end{tabular}

Fuente: elaborado en base a Jorrat (2008), pp. 12-13.

Cuadro 2. Indicadores descriptivos de movilidad. AMBA, 2004

\begin{tabular}{|l|l|}
\hline \multicolumn{2}{|c|}{ Indicadores } \\
\hline Inmovilidad & $36,1 \%$ \\
\hline Movilidad total & $63,9 \%$ \\
\hline Movilidad ascendente & $28,5 \%$ \\
\hline Movilidad descendente & $19,5 \%$ \\
\hline Movilidad circulatoria & $48,7 \%$ \\
\hline Movilidad estructural & $15,3 \%$ \\
\hline
\end{tabular}

Fuente: elaborado en base a Cuadro 1.

Una primera aproximación a la lectura de esta tabla sería la siguiente: tomemos la diagonal principal. Nos estaría indicando la proporción de sujetos que no han experimentado un cambio de posición entre t0 (generación de los padres) y t1 (generación de los hijos). Si calculamos la proporción de sujetos que se encuentran en esta diagonal (sobre el total de casos), podemos considerar a la misma como una medida de la «inmovilidad» o «estabilidad» de la tabla. En este caso la «inmovilidad» es de $36,1 \%$. El complemento a $100 \%$ cuantificaría la «movilidad» en la tabla $(63,9 \%)$.

A continuación, podemos calcular las proporciones por «encima» y por «debajo» de la diagonal, lo cual brindaría una noción de las movilidades ascendentes (por encima de la diagonal) y descendentes (por debajo de la diagonal): en este caso $60 \%$ y $40 \%$, respectivamente. Esta descripción hace referencia a lo que ciertos autores (Goldthorpe et al. 1987) llaman movilidad «de facto». 
Este problema también ha sido abordado en torno a los conceptos de movilidad total, estructural y circulatoria, donde la movilidad total es igual a la suma de las otras dos. La llamada movilidad «estructural» se refiere a la movilidad mínima permitida por los marginales de la tabla. El objetivo, en este caso, es cuantificar algo así como una movilidad «técnica» que es efecto de los totales muestrales, de la distribución de la tabla y que no guardaría relación con la movilidad «realmente existente». Lo que se hace, entonces, es «aislar» el efecto que esta tiene sobre la «movilidad total». La diferencia entre ambas sería la «movilidad real».

es decir que,

$$
\mathrm{M}_{\text {tot }}=M_{\text {estruct }}+M_{\text {circ }}
$$

$$
\mathrm{M}_{\text {real }}=M_{\text {tot }}-M_{\text {estruct }}
$$

La movilidad estructural se obtiene como la diferencia entre el total muestral y la suma de las menores de las frecuencias marginales vinculadas a cada celda de la diagonal principal: en nuestro caso: $1677-(192+164+416+276+373)=256$. Esto daría una aproximación a la «máxima inmovilidad» permitida por las frecuencias marginales del cuadro. La diferencia de este valor con el total muestral señalaría la mínima movilidad que existiría en el cuadro, independientemente del régimen de movilidad existente en la sociedad. Sería un nivel de movilidad inescapable, que deviene de de la propia distribución de la tabla (Jorrat 2005).

La movilidad, vista desde esta dimensión quedaría definida con los siguientes valores: 48,7\% (movilidad circulatoria) y 15,3\% (movilidad estructural). Es decir, se observa un predominio de la movilidad «pura» $\mathrm{o}$ «circulatoria».

El problema con todas estas medidas radica en que se encuentran sumamente afectadas por los valores marginales de los cuadros (tal y como se desprende de su forma de cálculo). Volveremos con más detalles sobre este punto más adelante. Es por ello que muchos investigadores han intentado desarrollar medidas que logren controlar el efecto de los marginales y lograr con ello cuantificar la movilidad «real».

\section{1. (In)movilidad social y $Q$ de Joule}

Una primera estrategia en este sentido es el índice de «movilidad/estabilidad» (I), el cual consiste simplemente en el cociente entre las frecuencias observadas en la tabla y las frecuencias esperadas bajo el supuesto de independencia estadística. De esta forma se expresa la movilidad observada como proporción de la esperada ${ }^{6}$. En todas aquellas celdas en que el índice sea mayor

${ }^{6}$ Lo interesante en la «historia» de este coeficiente es que fue elaborado por tres investigadores que llegaron por vías diferentes y de forma independiente, a conclusiones coincidentes en torno a los problemas que surgen en la lectura de matrices de movilidad y construyeron una herramienta similar.

EMPIRIA. Revista de Metodología de Ciencias Sociales. N. ${ }^{\circ} 22$, julio-diciembre, 2011, pp. 67-90. ISSN: $1139-5737$ 
que 1 , se tratará de celdas en las que la movilidad observada es mayor que la esperada y viceversa.

El primer paso, entonces es calcular la movilidad esperada bajo «independencia estadística», lo cual se realiza mediante la siguiente fórmula, basada en el teorema de la «probabilidad compuesta» (que afirma que la ocurrencia simultánea de dos o más sucesos independientes es igual al producto de sus probabilidades de ocurrencia por separado):

$$
\mathrm{fe}_{\mathrm{ij}}=\mathrm{n}_{\mathrm{i}} \mathrm{n}_{\mathrm{j}}
$$

Luego, se calcula la relación entre la frecuencia observada y la esperada:

$$
\mathrm{I}=\frac{\mathrm{fo}_{\mathrm{ij}}}{\mathrm{fe}_{\mathrm{ij}}}
$$

Si lo calculamos para la tabla 2, obtenemos los siguientes resultados:

Cuadro 3. Índice de «movilidad/estabilidad» (I). Argentina, 2003-04

\begin{tabular}{|l|c|c|c|c|c|}
\hline \multirow{2}{*}{\multicolumn{1}{|c|}{ Destino }} & \multicolumn{5}{|c|}{ Origen } \\
\cline { 2 - 6 } & $\begin{array}{c}\text { Clase de } \\
\text { Servicios }\end{array}$ & $\begin{array}{c}\text { No } \\
\text { Manuales }\end{array}$ & $\begin{array}{c}\text { Pequeña } \\
\text { burguesía }\end{array}$ & $\begin{array}{c}\text { Manuales } \\
\text { calificados }\end{array}$ & $\begin{array}{c}\text { Manuales } \\
\text { no } \\
\text { calificados }\end{array}$ \\
\hline Clase de Servicios & 2,72 & 1,42 & 0,91 & 0,68 & 0,47 \\
\hline No Manuales & 1,28 & 1,66 & 0,84 & 1,22 & 0,59 \\
\hline Pequeña burguesía & 0,80 & 0,91 & 1,50 & 0,71 & 0,82 \\
\hline Manuales calificados & 0,57 & 0,82 & 0,88 & 1,49 & 0,94 \\
\hline Manuales no calificados & 0,19 & 0,30 & 0,75 & 0,93 & 2,00 \\
\hline
\end{tabular}

Fuente: elaboración propia en base a Cuadro 1.

Como se ve, los mayores valores del índice se presentan en la diagonal principal, lo cual confirma que se trata de una tabla con «baja movilidad». A su vez, existen tres transiciones con valores significativamente superiores a la unidad: la transición de la «clase de servicios (padre)/ no manuales (hijos)»: 1,28; la de «manuales calificados (padre)/ no manuales (hijos)»: 1,22 y la de de «no manuales (padre)/ clase de servicios (hijos)» presenta el valor más elevado $(1,42)$.

Este coeficiente ha sido criticado dado que depende sobremanera del supuesto de independencia estadística, el cual es demasiado rígido e «irreal». Volveremos sobre esto más adelante.

Además, en tanto las frecuencias esperadas se calculan mediante el producto de los marginales de las distribuciones, este índice no soluciona el problema de la dependencia de los marginales (problema que estaba presente en los abordajes descriptivos). Esto implica, por ejemplo, que si tuviéramos dos tablas 
con diferentes marginales, pero similares asociaciones entre orígenes y destinos, daría como resultado índices de movilidad distintos (Hout 1985).

Por esta razón, se Andrea Tyree (1973) propuso la construcción de un índice basado en el coeficiente Q de Joule. Este tendría la ventaja de trabajar con valores no tan afectados por los marginales de las tablas (o al menos afectados indirectamente), dado que opera sobre la base de los productos cruzados de una distribución. A su vez, este índice brinda la ventaja de tener un supuesto de «asociación perfecta» mucho menos rígido que la que brinda el índice de inmovilidad. Volveremos sobre este punto.

En el caso que se quiera trabajar con una tabla $\mathrm{n} \times \mathrm{n}$, debe «reducirse» la misma a una distribución 2 x 2. Según Tyree esto se logra de forma directa con la siguiente fórmula:

$$
\mathrm{q}=\frac{\mathrm{n}_{\mathrm{ij}}\left(\mathrm{N}-\mathrm{n}_{\mathrm{i} .}-\mathrm{n}_{\mathrm{j} .}+\mathrm{n}_{\mathrm{ij}}\right)-\left(\mathrm{n}_{\mathrm{j}}-\mathrm{n}_{\mathrm{ij}}\right)\left(\mathrm{n}_{\mathrm{i} .}-\mathrm{n}_{\mathrm{ij}}\right)}{\mathrm{n}_{\mathrm{ij}}\left(\mathrm{N}-\mathrm{n}_{\mathrm{i} .}-\mathrm{n}_{\mathrm{j} .}+\mathrm{n}_{\mathrm{ij}}\right)+\left(\mathrm{n}_{\mathrm{j} j}-\mathrm{n}_{\mathrm{ij}}\right)\left(\mathrm{n}_{\mathrm{i} .}-\mathrm{n}_{\mathrm{ij}}\right)}
$$

Este coeficiente tiene cumpliría según la autora dos condiciones básicas para un «buen coeficiente»: a) valores extremos de +1 y -1 correspondiendo a los valores de asociación perfecta positiva y negativa, respectivamente (estandarización) y b) valor 0 para el caso de «independencia estadística» o ausencia de relación (Tyree 1973: 583).

Si aplicamos la fórmula propuesta a nuestra distribución original obtenemos la siguiente matriz de asociación.

Cuadro 4. Índice basado en Q de Joule. Argentina, 2003-04

\begin{tabular}{|l|c|c|c|c|c|}
\hline \multirow{2}{*}{\multicolumn{1}{|c|}{ Destino }} & \multicolumn{5}{|c|}{ Origen } \\
\cline { 2 - 6 } & $\begin{array}{c}\text { Clase de } \\
\text { Servicios }\end{array}$ & $\begin{array}{c}\text { No } \\
\text { Manuales }\end{array}$ & $\begin{array}{c}\text { Pequeña } \\
\text { burguesía }\end{array}$ & $\begin{array}{c}\text { Manuales } \\
\text { calificados }\end{array}$ & $\begin{array}{c}\text { Manuales } \\
\text { no } \\
\text { calificados }\end{array}$ \\
\hline Clase de Servicios & 0,67 & 0,23 & $-0,08$ & $-0,27$ & $-0,48$ \\
\hline No Manuales & 0,19 & 0,39 & $-0,15$ & 0,18 & $-0,39$ \\
\hline Pequeña burguesía & $-0,16$ & $-0,07$ & 0,42 & $-0,27$ & $-0,17$ \\
\hline Manuales calificados & $-0,34$ & $-0,13$ & $-0,10$ & 0,34 & $-0,05$ \\
\hline Manuales no calificados & $-0,76$ & $-0,63$ & $-0,24$ & $-0,06$ & 0,64 \\
\hline
\end{tabular}

Fuente: elaboración propia en base a Cuadro 1.

Al observar el cuadro 4 no se observan grandes diferencias en relación al índice de «movilidad/ estabilidad» (I): aparecen valores elevados en la diagonal principal, lo cual vuelve a evidenciar la pauta de «rigidez»o «herencia» en la movilidad social. 
Ahora bien, la principal desventaja de ambos coeficientes es que se basan sobre el binomio conceptual «independencia estadística/ asociación perfecta». Para ello comparan las distribuciones observadas con alguna frecuencia esperada que puede ser el producto de los marginales de la tabla (índice de inmovilidad) o el producto cruzado de las celdas interiores de la tabla (Q de Joule).

El problema en este punto es, digámoslo así, «teórico- metodológico»: sea que tratemos con matrices de movilidad intergeneracional o individuales, resulta difícil sostener en términos teóricos que la posición en la estructura social de un sujeto no tiene relación (es decir, es independiente estadísticamente) con a) la posición de sus progenitores y/ o b) su propia posición en la misma estructura en un momento anterior.

Si uno llevara al extremo este supuesto, el mismo equivale a suponer que la conformación y el movimiento de una estructura social determinada (esto es, la distribución de los sujetos en sus diferentes fracciones y capas) en un momento determinado, es independiente de su conformación en momentos anteriores. La estructura social, sus movimientos y las formas que asumen sus cambios serían, de esta manera, ininteligibles en tanto la afirmación anterior es equivalente a sostener que los movimientos de la estructura social resultan de una pura contingencia. Este punto queda claro en la cita de Gino Germani: el supuesto conceptual detrás del índice de inmovilidad es que las posiciones en el momento $\mathrm{t} 1$ se distribuyen al azar entre todos los sujetos, sin importar su origen, es decir, la posición que ocupaban en momentos anteriores.

Si hubiera que plantearlo en los términos clásicos del análisis estadístico, podríamos decir que la hipótesis nula $\left(\mathrm{H}_{0}\right)$ propone que la posición actual no depende de la anterior, mientras que la hipótesis alternativa $\left(\mathrm{H}_{1}\right)$ afirma lo contrario. Lo que se buscaría en el caso de estos índices sería no rechazar $\mathrm{H}_{0}$. Se explora, en realidad, la bondad de ajuste del modelo. Tal y como afirma Jorrat (2008: 24): «... el modelo de independencia nunca produce un buen ajuste: los destinos de alguna manera dependen de los orígenes.»

\section{MODELOS LOG-LINEALES}

Una manera de superar estas limitaciones ha sido buscada en la utilización de los modelos log-lineales, los cuales han representado un salto cualitativo en el análisis de tablas de movilidad. En efecto, desde la aparición del texto de Hauser (1978), los modelos log-lineales han sido utilizados profusamente en los análisis de movilidad social (por ejemplo, Echeverría Zabalza 1999). En Argentina, Kessler y Espinoza (2007) y Jorrat (2001, 2008), por nombrar algunos de los más recientes, han utilizado los modelos log-lineales para realizar el análisis de las tablas de movilidad.

La base del análisis es un modelo lineal-logarítmico que se propone predecir el valor de cada una de las casillas de una tabla de movilidad como la sumatoria de tres efectos: el efecto de pertenecer a la categoría de las filas $\left(\mathrm{f}_{\mathrm{i}}\right)$, el efecto de 
pertenecer a la categoría de las columnas (f $)$ y el efecto de la interacción entre las variables de las filas y columnas $\left(\mathrm{f}_{\mathrm{ij}}\right)$. Más precisamente se considera que cada una de las frecuencias en las casillas son el resultado de la sumatoria del logaritmo de estos tres efectos más la media aritmética del logaritmo de las frecuencias de cada una de las casillas de la tabla:

$$
\ln \left(\mathrm{f}_{i j}\right)=u+\ln \left(\mathrm{f}_{i}\right)+\ln \left(\mathrm{f}_{j}\right)+\ln \left(\mathrm{f}_{i j}\right)
$$

Si este razonamiento se generaliza para todas las celdas de la tabla resulta posible, entonces, estimar un modelo (o varios) que controlen uno (o varios) de los «efectos» en las celdas de la tabla de movilidad. Se comparan, entonces, el modelo «saturado» (el que contiene todos los efectos) con el modelo «estimado» y se evalúa la «bondad de ajuste» del modelo estimado.

Cuadro 5. Esquematización de modelos de «cuasi-independencia» y «esquina acotada»

\begin{tabular}{|l|c|c|c|c|c|}
\hline \multirow{2}{*}{\multicolumn{1}{c|}{ Destino }} & \multicolumn{5}{c|}{ Origen } \\
\cline { 2 - 6 } & $\begin{array}{c}\text { Clase de } \\
\text { Servicios }\end{array}$ & $\begin{array}{c}\text { No } \\
\text { Manuales }\end{array}$ & $\begin{array}{c}\text { Pequeña } \\
\text { burguesía }\end{array}$ & $\begin{array}{c}\text { Manuales } \\
\text { calificados }\end{array}$ & $\begin{array}{c}\text { Manuales } \\
\text { no } \\
\text { calificados }\end{array}$ \\
\hline Clase de Servicios & 1 & 2 & 3 & 3 & 3 \\
\hline No Manuales & 2 & 1 & 3 & 3 & 3 \\
\hline Pequeña burguesía & 3 & 3 & 1 & 3 & 3 \\
\hline Manuales calificados & 3 & 3 & 3 & 1 & 3 \\
\hline Manuales no calificados & 3 & 3 & 3 & 3 & 1 \\
\hline
\end{tabular}

Por ejemplo, si en el cuadro anterior identificamos la diagonal principal, resulta posible verificar que si se estima un modelo sin los efectos de la misma, se obtiene un modelo de «cuasi-independencia». Lo que se busca en el mismo es evaluar la «independencia estadística» que se verifica por fuera de esta diagonal (Kessler y Espinoza 2007).

De igual manera, a partir de este esquema podría estimarse un modelo de independencia estadística «a secas», simplemente controlando los efectos (en todas las celdas) del efecto conjunto (la interacción) de las variables de fila y columna: $\operatorname{los} \ln \left(\mathrm{f}_{\mathrm{ij}}\right)$ de la ecuación.

Los estudios más recientes sobre movilidad social en Argentina, han utilizado diversos modelos controlando diversos efectos en las tablas de movilidad. Jorrat $(2001,2008)$ y Kessler y Espinoza (2007) hacen especial uso de un modelo: el llamado de «esquina no manual acotada». En efecto, debido a las elevadas diferencias que se verifican en las transiciones entre los estratos «no manuales», intentan estimar como se comporta la «independencia estadística» en el resto de la tabla, si se controla el efecto de la movilidad existente el interior de los «no manuales». En el ejemplo que analizamos, equivaldría a estimar un 
modelo controlando las celdas numeradas con «2» en el cuadro 5. Presentamos una evaluación de este modelo en la siguiente sección.

Sin embargo, y pese a las ventajas de las técnicas log-lineales, existen limitaciones que afectan esta herramienta de análisis. De esta forma, intentaremos mostrar que los modelos log-lineales no resultan ser cualitativamente diferentes de los índices basados en el supuesto de independencia estadística. Volveremos sobre este punto sobre el final del trabajo.

\section{Elementos para la construcción de un índice de movilidad social: cadenas de Markov}

Consideramos que las cadenas de Markov pueden representar un instrumento de suma utilidad para intentar «modelizar» distintos patrones de movilidad social. Trataremos de aportar algunos elementos metodológicos que apunten a un nivel descriptivo del problema en cuestión: particularmente, la construcción de un índice de movilidad.

Constituyen una herramienta para analizar el comportamiento de ciertos procesos estocásticos, es decir, procesos que no respetan una pauta determinista en su forma de evolución, en su sucesión de estados a lo largo del tiempo. Más estrictamente un proceso estocástico puede definirse como un conjunto de variables indexadas de tipo $\mathrm{X}_{t}$, dónde $t$ asume valores en algún conjunto T. A los efectos de este trabajo $\mathrm{T}$ tomará valores discretos tal que $\mathrm{T}=1,2,3,4 \ldots \mathrm{n}$.

Una cadena de Markov representa un sistema que varía su estado a lo largo del tiempo y dónde cada cambio constituye una transición del sistema. Dichos cambios, tal y como se ha mencionado, no responden a una pauta determinista por lo cual no son «predecibles». Si es posible, en cambio, conocer las probabilidades de transición entre un estado y otro del sistema. Esto es, la probabilidad de un estado futuro en función de los estados anteriores.

Formalmente, para definir una cadena de Markov, son necesarios los siguientes elementos:

a) un conjunto de estados del sistema

b) una definición de transición

c) una ley de probabilidad condicional, que defina la probabilidad del nuevo estado en función de los estados anteriores

Se trata entonces, de un conjunto de variables indexadas en $t$, donde $t$ denota intervalos temporales significativos al fenómeno estudiado (en el caso concreto del análisis de la movilidad social, podría considerarse una generación). Los posibles valores de $\mathrm{X}_{\mathrm{t}}$ se toman de un conjunto de categorías mutuamente excluyentes denominadas estados del sistema. Por tratarse de un sistema estocástico no se conocerá con certeza el estado de un sistema en el momento $t$, pero si las probabilidades asociadas a cada uno de los estados. Para el caso de las cadenas de Markov debe tenerse en cuenta que dada la probabilidad condicional de 
un estado del sistema, su comportamiento futuro depende solamente del momento actual y no del comportamiento pasado del sistema:

$$
p\left(X_{t+1}=j / X_{0}, X_{1}, \ldots X_{t}\right)=p\left(X_{t+1}=j / X_{t}\right)
$$

lo que constituye la llamada «propiedad de Markov».

La base de la construcción de una cadena de Markov son las llamadas «probabilidades de transición». Una probabilidad de transición entre dos estados de un sistema a lo largo de dos instantes de tiempo, es la probabilidad condicionada de situarse en un estado determinado, habiéndose encontrado en otro estado en un instante de tiempo anterior. De esta forma, a partir de la cuantificación de la totalidad de las probabilidades de transición de los elementos del sistema a cada uno de los estados, puede construirse una «matriz de transición». Queda claro, entonces, que una matriz de transición tiene una estructura idéntica a una matriz de movilidad.

Una matriz de transición es una matriz cuadrada, donde cada fila y cada columna corresponden a uno de los estados posibles del sistema y los elementos de la matriz representan la probabilidad de que el estado próximo sea el de la columna, si el estado actual es el correspondiente a la fila.

Como el sistema debe evolucionar en $t$ a alguno de los estados siguientes, las probabilidades de transición cumplirán la propiedad siguiente:

$$
\sum_{j}^{n}=1 p_{i j}=1
$$

Además, por la misma definición de probabilidad, cada una de estas probabilidades ha de ser no negativas:

$$
\mathrm{p}_{\mathrm{ij}} \geq 0
$$

Cuando las $\mathrm{p}_{\mathrm{ij}}$ cumplen con las propiedades anteriores, la matriz $\mathrm{P}$ es una matriz estocástica: la suma de los valores de las filas será igual a 1 (mientras que la de las columnas no tendrá ninguna probabilidad especial).

La diferencia (y su principal ventaja) con los índices que hemos expuesto anteriormente radica, justamente, en esa matriz de transición. Mientras que el índice de (in)movilidad (I) y el $Q$ de Joule trabajan sobre el supuesto de «independencia estadística», un índice construido sobre una cadena de Markov permite estimar frecuencias esperadas, pero trabajando sin un supuesto teórico previo tan rígido, sino sobre la base de una distribución real y observada.

También se logra flexibilizar el supuesto complementario al de «independencia estadística»: el de asociación perfecta. Como todos sabemos, buena parte de los coeficientes de asociación típicamente utilizados, parten de algún tipo de definición de lo que significa «asociación perfecta». En el caso de los coeficientes basados en el chi cuadrado (basado en una lógica similar al analizad índice de inmovilidad social), esta definición asume su posición más extrema: aso- 
ciación perfecta implica correspondencia absoluta entre los valores de ambas variables.

Por ejemplo, en una tabla de 2 × 2, la asociación perfecta quedaría definida concentrando la totalidad de los casos en una de las diagonales:

\begin{tabular}{llllll}
\hline & $\mathbf{X}$ & & $\mathbf{Y}$ & \\
\cline { 2 - 5 } & & $\mathbf{c}$ & & $\mathbf{d}$ & \\
\hline A & & & $\mathrm{X}$ & 0 \\
B & & 0 & $\mathrm{X}$ \\
\hline
\end{tabular}

(dónde las X mayúsculas indican mayor concentración de frecuencias y los 0 ausencia absoluta de casos)

En el caso del coeficiente Q de Joule este supuesto es un poco más flexible en tanto no supone la concentración de los casos en la diagonal, sino que podría presentar un valor cercano a 1 en una distribución como la siguiente:

\begin{tabular}{lllll}
\hline & $\mathbf{X}$ & \multicolumn{3}{c}{$\mathbf{Y}$} \\
\cline { 2 - 5 } & $\mathbf{c}$ & & $\mathbf{d}$ & \\
\hline $\mathbf{A}$ & & & $\mathrm{X}$ & $\mathrm{X}$ \\
$\mathbf{B}$ & & $\mathrm{X}$ & $\mathrm{X}$ \\
\hline
\end{tabular}

(donde las X mayúsculas indican mayor concentración de frecuencias y las x minúsculas, menor concentración de casos)

Lo interesante de las cadenas de Markov es que pueden escapar a estos supuestos y trabajar sobre inferencias realizadas sobre los mismos datos observados. En términos teóricos, el supuesto de independencia estadística (repitámoslo una vez más) equivale a suponer que la posición en la estructura social en un momento dado no guarda relación con la posición ocupada en un momento (o una generación) anterior. La distribución de posiciones sociales sería aleatoria e independiente de los orígenes.

Ahora bien, un índice de movilidad basados en las cadenas de Markov (tal y como proponemos en este trabajo) sería equivalente a suponer que la movilidad social (expresada en las matrices de transición) se mantiene constante ${ }^{7}$ (es decir, las probabilidades de transición se mantienen constantes) durante un período de tiempo t.

De esta forma se estiman los movimientos de la población a partir de una

${ }^{7}$ También existen modelos que consideran la posibilidad de utilizar distintas matrices de transición en diferentes momentos del tiempo. Es decir que la los flujos de población en la movilidad social no son «constantes». En nuestro caso, consideramos más apropiado trabajar con esa forma «simple», dado que a) existe escasa disponibilidad de datos sobre la movilidad «intergeneracional»; b) estamos estimando la movilidad en períodos relativamente cortos de tiempo y solamente en dos momentos sucesivos. 
distribución dada. Como consecuencia del paso del tiempo, la población se irá moviendo de «estado» a «estado», siguiendo las pautas determinadas por las distintas probabilidades de transición (que se asumen constantes) con lo cual irá modificándose la matriz de transición.

Por otro lado, y como una ventaja adicional, al «estimar» las frecuencias esperadas, no sobre los marginales de la tabla, sino sobre las frecuencias relativas de la matriz de transición, los índices construidos no estarán sujetos a la dependencia de esos marginales. Razón por la cual, si encontrásemos dos tablas de movilidad donde existe en cada una de ellas un grado similar de asociación entre los dos estados de la movilidad social; pero, no obstante, presenta diferentes distribuciones absolutas es de suponer que obtendríamos índices similares.

Ahora bien, este instrumental no está exento de algunos supuestos propios que deben ser explicitados. El más importante de ellos se refiere a la homogeneidad temporal de la población. Esto significa que las probabilidades de transición definidas son constantes en el tiempo. Es decir, dichas probabilidades dependen solamente de la cantidad de períodos transcurridos, no variando entre cada uno de los períodos ${ }^{8}$.

A diferencia de otros estudios, donde se dispone de una sucesión de tablas de movilidad y donde el mayor problema es la estimación de una matriz de transición única a partir de esa multiplicidad de tablas ${ }^{9}$, en nuestro caso contamos solamente con una tabla de movilidad. Es decir que no nos enfrentamos con el problema de lograr una matriz de transición que pueda reflejar los cambios sucesivos en el tiempo que se expresan en cada una de las tablas de movilidad parciales. Pero esta posibilidad aparece como posibilidad (al menos latente) si fuera posible incorporar información histórica relativa a la movilidad social, es decir, matrices de movilidad de períodos diferentes y que cumplieran la condición de ser comparables entre sí (condición que en caso de la disponibilidad de datos en Argentina resulta, por lo menos, remota).

Veamos, ahora, como quedaría formulado nuestro nuevo «modelo», en función de la distribución que hemos venido analizando hasta aquí. A partir de los datos oportunamente expuestos obtendríamos nuestro primer insumo para la matriz de transición:

${ }^{8}$ Existen una serie de técnicas para flexibilizar este supuesto, pero requieren de cálculos más complejos. No los incluimos en este trabajo, en tanto todavía estamos evaluando la factibilidad de aplicar estos modelos al problema de la «movilidad social». Un resumen de estos métodos y una aplicación concreta a la movilidad territorial de la población (migración) española puede verse en Hierro Franco y Guijarro Garvi (2006).

${ }^{9}$ Un ejemplo puede verse en Hierro Franco y Guijarro Garvi (2006). Estos autores intentan estimar una matriz de transición a partir de los datos de movilidad migratoria para distintas clases de ciudades españolas entre los años 1990-2003. Deben trabajar a partir de 13 tablas de movilidad para lograr una única matriz de transición que les permita calcular la probabilidad que un individuo se mueva de la clase de municipio «A» a la clase «B».

EMPIRIA. Revista de Metodología de Ciencias Sociales. N. ${ }^{\circ}$ 22, julio-diciembre, 2011, pp. 67-90. ISSN: $1139-5737$ 
Cuadro 6. Matriz de transición observada. Movilidad desde la ocupación o clase de los padres hacia la de los encuestados. Ambos sexos (relativos, porcentaje sobre columna). Argentina, 2003-04

\begin{tabular}{|l|c|c|c|c|c|c|}
\hline \multirow{2}{*}{\multicolumn{1}{|c|}{ Destino }} & \multicolumn{6}{|c|}{ Origen } \\
\cline { 2 - 7 } & $\begin{array}{c}\text { Clase de } \\
\text { Servicios }\end{array}$ & $\begin{array}{c}\text { No } \\
\text { Manuales }\end{array}$ & $\begin{array}{c}\text { Pequeña } \\
\text { burguesía }\end{array}$ & $\begin{array}{c}\text { Manuales } \\
\text { calificados }\end{array}$ & $\begin{array}{c}\text { Manuales } \\
\text { no } \\
\text { calificados }\end{array}$ & Total \\
\hline Clase de Servicios & $37,5 \%$ & $19,5 \%$ & $12,5 \%$ & $9,4 \%$ & $6,4 \%$ & $13,8 \%$ \\
\hline No Manuales & $29,1 \%$ & $37,8 \%$ & $19,1 \%$ & $27,7 \%$ & $13,4 \%$ & $22,7 \%$ \\
\hline Pequeña burguesía & $19,8 \%$ & $22,6 \%$ & $37,2 \%$ & $17,6 \%$ & $20,2 \%$ & $24,8 \%$ \\
\hline Manuales calificados & $9,4 \%$ & $13,4 \%$ & $14,5 \%$ & $24,5 \%$ & $15,5 \%$ & $16,5 \%$ \\
\hline Manuales no calificados & $4,2 \%$ & $6,7 \%$ & $16,7 \%$ & $20,8 \%$ & $44,5 \%$ & $22,2 \%$ \\
\hline Total & $100,0 \%$ & $100,0 \%$ & $100,0 \%$ & $100,0 \%$ & $100,0 \%$ & $100,0 \%$ \\
\hline
\end{tabular}

Fuente: elaborado en base a Cuadro 1.

A partir de esta tabla intentaremos estimar una matriz de transición. El ejercicio que proponemos es el siguiente. Disponemos de una matriz de movilidad, en lugar de preguntarnos cuál sería la distribución si ambas variables (origen y destino) fueran estadísticamente independientes, nos preguntamos cuál sería la distribución si esa matriz de movilidad fuera constante en el tiempo (es decir, constante durante una generación). Para ello simplemente es necesario elevar esa matriz al cuadrado (es decir, multiplicarla por sí misma).

Cuadro 7. Matriz de transición estimada en base a supuesto de Markov

\begin{tabular}{|l|c|c|c|c|c|}
\hline \multirow{2}{*}{\multicolumn{1}{|c|}{ Destino }} & \multicolumn{5}{|c|}{ Origen } \\
\cline { 2 - 6 } & $\begin{array}{c}\text { Clase de } \\
\text { Servicios }\end{array}$ & $\begin{array}{c}\text { No } \\
\text { Manuales }\end{array}$ & $\begin{array}{c}\text { Pequeña } \\
\text { burguesía }\end{array}$ & $\begin{array}{c}\text { Manuales } \\
\text { calificados }\end{array}$ & $\begin{array}{c}\text { Manuales } \\
\text { no } \\
\text { calificados }\end{array}$ \\
\hline Clase de Servicios & 0,233 & 0,192 & 0,155 & 0,148 & 0,118 \\
\hline No Manuales & 0,289 & 0,289 & 0,242 & 0,261 & 0,210 \\
\hline Pequeña burguesía & 0,239 & 0,245 & 0,266 & 0,232 & 0,235 \\
\hline Manuales calificados & 0,133 & 0,145 & 0,153 & 0,164 & 0,160 \\
\hline Manuales no calificados & 0,107 & 0,129 & 0,185 & 0,195 & 0,276 \\
\hline
\end{tabular}

Fuente: elaboración propia en base a Cuadro 5.

Luego, a partir de esta podemos calcular las frecuencias esperadas en cada una de las celdas a partir de la distribución relativa en el sentido de las columnas (cuadro 6): 
Cuadro 8. Matriz de frecuencias estimadas bajo supuesto de Markov. Argentina, 2003-04

\begin{tabular}{|l|r|r|r|r|r|}
\hline \multirow{2}{*}{\multicolumn{1}{|c|}{ Destino }} & \multicolumn{5}{|c|}{ Origen } \\
\cline { 2 - 6 } & $\begin{array}{c}\text { Clase de } \\
\text { Servicios }\end{array}$ & $\begin{array}{c}\text { No } \\
\text { Manuales }\end{array}$ & $\begin{array}{c}\text { Pequeña } \\
\text { burguesía }\end{array}$ & $\begin{array}{c}\text { Manuales } \\
\text { calificados }\end{array}$ & $\begin{array}{c}\text { Manuales } \\
\text { no } \\
\text { calificados }\end{array}$ \\
\hline Clase de Servicios & 44,86 & 31,48 & 77,00 & 59,63 & 49,74 \\
\hline No Manuales & 55,41 & 47,38 & 120,36 & 105,61 & 88,38 \\
\hline Pequeña burguesía & 45,83 & 40,22 & 131,98 & 93,66 & 98,81 \\
\hline Manuales calificados & 25,44 & 23,77 & 75,89 & 66,15 & 67,29 \\
\hline Manuales no calificados & 20,46 & 21,15 & 91,77 & 78,95 & 115,78 \\
\hline
\end{tabular}

Fuente: elaboración propia en base a Cuadro 6.

Por último, calculando la razón entre las frecuencias observadas y las esperadas bajo el supuesto de Markov obtenemos nuestro índice de movilidad social. La lógica es la misma que la utilizada en el índice de inmovilidad: la movilidad observada se expresa como una proporción respecto de la movilidad esperada.

Cuadro 9. Índice de movilidad social bajo supuesto de Markov. Argentina, 2003-04.

\begin{tabular}{|l|c|c|c|c|c|}
\hline \multirow{2}{*}{\multicolumn{1}{c|}{ Destino }} & \multicolumn{5}{|c|}{ Origen } \\
\cline { 2 - 6 } & $\begin{array}{c}\text { Clase de } \\
\text { Servicios }\end{array}$ & $\begin{array}{c}\text { No } \\
\text { Manuales }\end{array}$ & $\begin{array}{c}\text { Pequeña } \\
\text { burguesía }\end{array}$ & $\begin{array}{c}\text { Manuales } \\
\text { calificados }\end{array}$ & $\begin{array}{c}\text { Manuales } \\
\text { no } \\
\text { calificados }\end{array}$ \\
\hline Clase de Servicios & 1,61 & 1,02 & 0,81 & 0,64 & 0,54 \\
\hline No Manuales & 1,01 & 1,31 & 0,79 & 1,06 & 0,63 \\
\hline Pequeña burguesía & 0,83 & 0,92 & 1,40 & 0,76 & 0,86 \\
\hline Manuales calificados & 0,71 & 0,93 & 0,95 & 1,50 & 0,97 \\
\hline Manuales no calificados & 0,39 & 0,52 & 0,90 & 1,06 & 1,62 \\
\hline
\end{tabular}

Fuente: elaboración propia en base a Cuadro 7.

Resulta interesante remarcar que en términos absolutos el índice construido no evidencia diferencias demasiado significativas en relación a los anteriores. En efecto, se observa que los mayores valores se concentran en la diagonal principal, por lo cual, se trata de una tabla que presenta elevados niveles de «estabilidad» $\mathrm{o}$ «inmovilidad». En particular, el pasaje de «clase de servicios (padre)/ manuales no calificados (hijo)» parece ser la categoría más fluctuante en esta estructura social: en los tres índices presenta valores elevados. Esto podría ser un indicador de la existencia de un proceso de «descenso» social. 
Sin embargo, si comparamos los resultados de cada una de las celdas de esta distribución con la que resulta de la confección del índice de «movilidad/ estabilidad», puede verse que existen algunas diferencia en la «captación» de la intensidad de los distintos estados de transición: las principal diferencia se da en la transición «no manuales (padre)/ clase de servicios (hijos)», donde el índice «movilidad/ estabilidad» (I) expresa un valor de 1,42 (es decir que marca una alta relación, en tanto la movilidad observada es un $42 \%$ mayor de la esperada); el índice de Markov, en cambio, presenta un valor bastante inferior $(1,02)$, lo cual expresa una escasa dependencia entre ambas posiciones. En la transición «clase de servicios (padre)/ no manuales (hijo)» se observa una diferencia similar: el índice de inmovilidad $(1,28)$ arroja valores elevados y el índice de Markov, valores casi cercanos a la independencia $(1,01)$.

Ahora bien, el índice de Markov parece captar con mayor sensibilidad las transiciones relacionadas con la movilidad descendente de «larga distancia»10: en efecto, los valores del índice de Markov resultan más elevados que los del de inmovilidad en las transiciones «clase de servicios (padre)/no calificados manuales (hijo)»y «no manuales (padre)/ no calificados manuales (hijo)».

Por último, aparecen algunas diferencias en la intensidad (no en la existencia) de la relación captada en las categorías extremas de la diagonal principal. En efecto, el índice de inmovilidad arroja valores mucho más elevados que el de Markov en la diagonal correspondiente a las categorías «clase de servicios (padre)/ clase de servicios (hijo)»y «no calificados manuales (padre)/ no calificados manuales (hijo)». Esto tiende a sugerir que el índice de Markov se ve menos afectado que el de inmovilidad por los valores de la diagonal principal.

Al verificar las diferencias entre el índice de Tyree ( $Q$ de Joule) y el construido sobre el supuesto de Markov también aparecen algunas diferencias. En todos los casos parece ser el índice basado en Markov el que presenta mayores sensibilidades a las siguientes transiciones: «clase de servicios (padre)/ calificados manuales (hijo)»; «cuenta propia (padre)/ clase de servicio (hijo)»; «cuenta propia (padre)/ no manuales (hijo)»; «calificados manuales (padre)/ clase de servicio (hijo)»; «no calificados manuales (padre)/ cuenta propia (hijo)».

En efecto, en todas estas, el índice de Markov arroja asociaciones mucho más fuertes que aquellas que muestra el índice Q de Joule. En cambio, en este caso no parecen encontrarse diferencias significativas en las asociaciones verificadas en la diagonal principal entre ambos índices.

Estas diferencias, obviamente, provienen de los diferentes supuestos teóricometodológicos sobre los que es posible operar si se trabaja con las herramientas provistas por las cadenas de Markov. En particular, con respecto al índice de «movilidad/inmovilidad» las diferencias provienen del hecho de que las frecuencias esperadas calculadas para cada uno son divergentes.

\footnotetext{
${ }^{10}$ Es decir, la movilidad social de carácter descendente que supera un casillero en la tabla de movilidad.
} 
Ahora bien, ¿cómo resumir algunas de las cuestiones que hemos discutido aquí? Podemos intentar una comparación del «ajuste» de algunos de los modelos mencionados en este trabajo a partir del estadístico ${ }^{11} \mathrm{G}^{2}$. Compararemos 4 modelos:

a) el de independencia estadística (en el cual se basan implícitamente el índice de inmovilidad y el de Tyree)

b) dos modelos basados en la utilización de modelos de análisis log-lineales: el de cuasi-independencia y el de esquinas acotadas

c) el modelo de frecuencias esperadas basadas en el supuesto de Markov

Además, incorporaremos los datos de un estudio anterior (Jorrat, 2001). El mismo fue realizado por el CEDOP de la Universidad de Buenos Aires y fue aplicado en el Área Metropolitana de Buenos Aires (AMBA) a encuestados de ambos sexos de 20 años y más.

Cabe una aclaración, sin embargo. Ambos estudios, además de las diferencias en la cobertura espacial y en las edades de los entrevistados, utilizan sistemas clasificatorios diferentes: el del 1995, utiliza un esquema de clasificación de cuatro clases («alto-no manual», «bajo-no manual», «alto-manual» y «bajomanual»), mientras que el de 2003-2004, utiliza el conocido esquema EGP ${ }^{12}$. Si bien según el autor, ambos esquemas no son «necesariamente incompatibles» (Jorrat 2001: 198), esto hace que los resultados de las estimaciones de los modelos no sean comparables entre ambos momentos del tiempo.

Es decir, no podremos comparar como cambia el ajuste de los modelos entre 1995 y 2004. Si, en cambio, es posible comparar el ajuste de los distintos modelos en cada momento del tiempo. Pese a esta limitación nos pareció interesante incorporar dos momentos temporales en el análisis, a los efectos de controlar, al menos en parte, ciertas «fluctuaciones» relacionadas al período histórico13. Hemos replicado los cálculos realizados sobre el estudio de 2003-04 al estudio de 2001 (se encuentran las tablas básicas en el anexo).

\footnotetext{
${ }^{11} \mathrm{El} \mathrm{G}$ (coeficiente de verosimilitud) se calcula como $\mathrm{G}^{2}=2 \sum_{\mathrm{ij}}^{\mathrm{fi}} \mathrm{x} \log \left(\mathrm{f}_{\mathrm{ij}} / \mathrm{F}_{\mathrm{ij}}\right)$. O sea, que compararemos las frecuencias observadas $\left(f_{i j}\right)$ y las esperadas $\left(F_{i j}\right)$ en estos 4 modelos.

${ }^{12}$ Esquema clasificatorio elaborado por Erikson, Goldthorpe y Portocarero. Cfr. Goldthorpe et al. 1987.

${ }^{13}$ Debe tenerse en cuenta que el año 2003 se corresponde con los momentos de «salida» de una crisis económica y social gigantesca en la Argentina, posterior a la salida de la paridad cambiaria 1 dólar=1 peso (convertibilidad). Como contrapartida, el año 1995 constituye un momento dónde la convertibilidad se encontraba consolidada.
} 
Cuadro 10. Evaluación de ajuste de modelos para el análisis de movilidad social. Argentina, 1995 y 2003-04

\begin{tabular}{|l|c|c|c|}
\hline Argentina 1995 & $\mathrm{G}^{2}$ & $\mathrm{p}$-valor & $\mathrm{gl}$ \\
\hline Independencia estadística & 332,5 & 0,00 & 9 \\
\hline Cuasi-independencia & 116,1 & 0,00 & 5 \\
\hline Modelo de las esquinas & 2,81 & 0,52 & 3 \\
\hline \multicolumn{4}{|l|}{} \\
\hline Markov & 142,8 & 0,00 & 9 \\
\hline
\end{tabular}

\begin{tabular}{|l|c|c|c|}
\hline Argentina 1995 & $\mathrm{G}^{2}$ & $\mathrm{p}$-valor & $\mathrm{gl}$ \\
\hline Independencia estadística & 340,4 & 0,000 & 16 \\
\hline Cuasi-independencia & 78,3 & 0,000 & 11 \\
\hline Modelo de las esquinas & 36,6 & 0,000 & 7 \\
\hline \multicolumn{4}{|l|}{} \\
\hline Markov & 161,84 & 0,000 & 16 \\
\hline
\end{tabular}

Fuente: ajustes de índice Markov elaborados en base a Cuadros 8 y 11; resto de ajustes tomados de Jorrat (2001) y Jorrat (2008).

Como puede notarse, además, existen diferencias en las distintas bondades de ajuste de cada uno de los modelos. En ambos años, es el de independencia estadística el que menor ajuste brinda. Tampoco el de cuasi-independencia logra un buen ajuste del modelo. Solamente el modelo de las esquinas es el que parece brindar mejores resultados, atendiendo al criterio de bondad de ajuste.

Ahora bien, ¿qué sucede con el índice de Markov? Si nos atenemos solamente al criterio de bondad de ajuste, cabría decir que este modelo presenta una mejora sustantiva, en ambos momentos, en relación al modelo de independencia. En relación al modelo de cuasi-independencia parece mostrar un valor de $\mathrm{G}^{2}$ cercano, pero deficiente en relación al modelo de las equinas. Sin embargo, si se incorporan otros criterios adicionales al cálculo de una bondad de ajuste, para analizar esta cuestión podemos arriesgar algunas reflexiones.

En primer lugar, cabe recordar que tanto el modelo de independencia como los basados en log-lineales siguen estando influidos en su cálculos por los valores de las frecuencias absolutas y los marginales (recordemos que se supone que la frecuencia de una celda es el resultado de la siguiente suma: el logaritmo del marginal de las filas, el logaritmo del marginal de las columnas y el logaritmo de la celda). De esta manera, el problema de las influencias de los marginales no aparece resuelto completamente (aunque se avanza en su resolución al incorporar el uso de logaritmos) en los modelos basados en técnicas log-lineales.

En segundo lugar, cabe problematizar la relación «costo-beneficio» que implica el uso de modelos basados en técnicas log-lineal. Si se observa el único modelo que «ajusta» a los datos (el modelo de las esquinas acotadas) puede ver- 
se la enorme pérdida de grados de libertad que supone. En efecto, para llegar a un modelo que «ajuste», se pierden 6 grados de libertad en 1995 (66,6\% del total) y 9 en 2003-04 (56,7\% del total). La ventaja del índice de Markov, resulta clara en este aspecto: no se pierden grados de libertad en la estimación de las frecuencias esperadas.

En último lugar, cabe recordar la posibilidad latente, explicitada más arriba, de realizar «estimaciones» de matrices de movilidad a partir de información histórica, es decir, a partir de la incorporación de matrices de movilidad de períodos diferentes.

Ahora bien, ambas cuestiones planteadas aquí refieren, por así decirlo, a la dimensión «técnica» del análisis. Si pensamos más allá de esta dimensión (sin excluirla, por supuesto) caben algunas reflexiones sobre los supuestos detrás de los diferentes modelos.

En términos estrictos, los tres modelos de análisis (índices de inmovilidad, Q de Joule y log-lineal) mantienen una característica común: el binomio conceptual «asociación/ independencia». Este carácter resulta claro en los primeros instrumentos: el índice de inmovilidad se basa en el cálculo de las frecuencias esperadas sobre la base del supuesto de independencia estadística perfecta. El índice basado en el Q de Joule también se basa en una noción de independencia estadística (aunque menos restrictiva). Los modelos log-lineales también buscan contrastar, en última instancia, una serie de frecuencias esperadas en base a un supuesto de independencia estadística: un supuesto de independencia acotada a ciertas regiones de la tabla de movilidad ${ }^{14}$, es cierto, pero un supuesto de independencia al fin.

Una prueba de esto es que puede realizarse el mismo test chi cuadrado de independencia estadística sobre la base de un modelo log-lineal: «ajustando» un modelo en el cual se eliminan los efectos de interacción entre las variables y manteniendo solamente los efectos de «fila y columna».

Por último, quizás cabría marcar, como una «hipótesis de lectura», una paradoja en el desarrollo de los modelos log-lineales. Recordemos que surgen como un intento de superar las herramientas de análisis existentes, básicamente, en función de la «rigidez conceptual» de éstas (la hipótesis de una distribución al azar de las posibilidades de movilidad social). En el intento de controlar los diversos efectos (marginales, movilidad estructural, etc.) y de «flexibilizar» el supuesto de la «distribución azarosa», pareciera haberse terminado priorizando la «bondad de ajuste» de los modelos a los datos. ¿En qué medida supone esto el retorno, en parte, al carácter descriptivo de las primeras herramientas de análisis de la movilidad social, basadas en el análisis de «entradas» y «salidas»?

${ }^{14}$ «El análisis log-lineal permite identificar zonas de densidad homogénea en que el origen ocupacional no influye sobre el destino. La independencia estadística que se verifica al interior de la zona refleja iguales oportunidades de movilidad para esas ocupaciones.» (Kessler y Espinoza 2007: 295)

EMPIRIA. Revista de Metodología de Ciencias Sociales. N. ${ }^{\circ} 22$, julio-diciembre, 2011, pp. 67-90. ISSN: $1139-5737$ 


\section{COMENTARIOS FINALES}

Hemos intentado reseñar algunos de los métodos habitualmente utilizados para realizar la lectura y el análisis de tablas de movilidad social. Repasamos algunos de los índices y metodologías más utilizadas, marcamos sus limitaciones y ventajas conceptuales (principalmente las derivadas del supuesto de «independencia estadística») y metodológicas (en función de la dependencia de los marginales).

Luego, intentamos exponer algunos elementos conceptuales para la construcción de un índice de movilidad que logre superar alguna(s) de esta(s) limitacion(es). La utilización de la teoría de las cadenas de Markov aparece como un elemento útil y fértil al respecto. Estas ventajas aparecen en la dimensión «técnica» (no dependencia de los marginales de la tabla, no utilización de supuestos de «asociación/ independencia»), pero también la dimensión conceptual: permite dar un rodeo a las implicancias conceptuales presentes en las herramientas basadas en los diversos supuestos de independencia estadística (total o acotada) y permite sostener y poner a prueba un supuesto de tipo diferente: en lugar de suponer que los destinos son independientes de los orígenes, puede utilizar el supuesto de la que dependencia de destinos y orígenes se mantiene constante durante determinado período de tiempo.

Solo queda remarcar el carácter tentativo y provisorio de estas consideraciones realizadas hasta aquí, esperando que puedan ser útiles como una introducción y un planteo de nuevas preguntas y problemas, más que de certezas.

\section{BIBLIOGRAFÍA}

Baltazar, E., Grillo Astudillo, S., y Alonso Malaver, C. (2003): «Movilidad social y transmisión de la pobreza en Bogotá», Economía y desarrollo, 2: disponible en www.fuac.edu.co/revista/I/I/seis.pdf. [consulta 08-07-2010]

BeCCARIA, J. (1978): «Una contribución al estudio de la movilidad social en la Argentina. Análisis de los resultados de una encuesta para el Gran Buenos Aires», Desarrollo Económico, 68, pp. 593-618.

ECHEVERRiA ZABAlZA, J. (1999): La movilidad social en España, 1940-1991, Madrid, Istmo.

GERMANI, G. (1965): «Apéndice II. La movilidad social en Argentina», Bendix, R. y Lipset, S. (1965): Movilidad social en la sociedad industrial, Buenos Aires, EUDEBA.

GoldTHORPE, J. et al. (1987): Social Mobility and Class Structure in Modern Britain, Oxford, Clarendon.

GolovanEVSKY, L. (2008): «Transmisión intergeneracional de la pobreza en la Argentina: una aproximación empírica», Estudios del Trabajo, 26, pp. 85-122.

Hierro Franco, M. y GuiJarRo Garvi, M. (2006): «Un estudio mediante cadenas de Markov de la dinámica de los movimientos migratorios interterritoriales en España (1990-2002) desde un planteamiento de estimación dinámico», Revista Asturiana de Economía, 35: disponible en www.revistaasturianadeeconomia.org/raepdf/35/ P145HIERRO.pdf. [consulta 14-08-2009] 
Hout, M. (1983): Mobility Tables. California, Sage.

JORRAT, J. R. (1986): «Las elecciones de 1983: ¿desviación o realineamiento?», Desarrollo Económico, 101, pp. 89-120.

JORRAT, J. R. (1995): Estratificación social y movilidad. Un estudio del Área Metropolitana de Buenos Aires, Tucumán, Ed. Univ. Tucumán.

JORRAT, J. R. (2005): «Aspectos descriptivos de la movilidad intergeneracional de clase en la Argentina: 2003- 2005», ponencia en $7^{\circ}$ Congreso Nacional de Estudios del Trabajo, Buenos Aires.

JORRAT, J. R. (2008): Exploraciones sobre movilidad de clase en Argentina: 20032004, IIGG Documento de trabajo n 52 , Instituto de investigaciones Gino Germani, Buenos Aires.

KESSLER, G. y ESPINOZA, V. (2007): «Movilidad social y trayectorias ocupacionales en Buenos Aires. Continuidades, rupturas y paradojas», Franco, R., León, A., Atria, R. (coord.) (2007): Estratificación y movilidad social en América Latina. Transformaciones estructurales en un cuarto de siglo, Santiago de Chile, LOM-CEPAL-GTZ.

Tyree, A. (1973): «Mobility ratios and association in mobility tables», Population Studies, 3, pp. 577-588.

\section{ANEXO ESTADÍSTICO}

Cuadro11. Movilidad desde la ocupación o clase de los padres hacia la de los encuestados. Ambos sexos (absolutos). AMBA, 1995

\begin{tabular}{|l|c|c|c|c|c|}
\hline \multirow{2}{*}{\multicolumn{1}{|c|}{ Destino }} & \multicolumn{5}{c|}{ Origen } \\
\cline { 2 - 6 } & $\begin{array}{c}\text { Alto no } \\
\text { manual }\end{array}$ & $\begin{array}{c}\text { Bajo no } \\
\text { manual }\end{array}$ & Alto manual & Bajo manual & Total \\
\hline Alto no manual & 91 & 107 & 75 & 53 & 326 \\
\hline Bajo no manual & 59 & 111 & 141 & 122 & 433 \\
\hline Alto manual & 27 & 76 & 190 & 190 & 483 \\
\hline Bajo manual & 11 & 53 & 154 & 309 & 527 \\
\hline Total & 188 & 347 & 560 & 674 & 1.769 \\
\hline
\end{tabular}

Fuente: elaborado en base a Jorrat (2001), p. 201. 
Cuadro 12. Matriz de transición observada. Movilidad desde la ocupación o clase de los padres hacia la de los encuestados. Ambos sexos (relativos, porcentaje sobre columna). AMBA, 1995

\begin{tabular}{|l|r|c|c|c|c|}
\hline \multirow{2}{*}{\multicolumn{2}{c|}{ Destino }} & \multicolumn{2}{c|}{ Origen } & \multirow{2}{*}{ Total } \\
\cline { 2 - 6 } & $\begin{array}{r}\text { Alto no } \\
\text { manual }\end{array}$ & $\begin{array}{c}\text { Bajo no } \\
\text { manual }\end{array}$ & Alto manual & Bajo manual & \multicolumn{2}{c|}{} \\
\hline Alto no manual & $48,4 \%$ & $30,8 \%$ & $13,4 \%$ & $7,9 \%$ & $18,4 \%$ \\
\hline Bajo no manual & $31,4 \%$ & $32,0 \%$ & $25,2 \%$ & $18,1 \%$ & $24,5 \%$ \\
\hline Alto manual & $14,4 \%$ & $21,9 \%$ & $33,9 \%$ & $28,2 \%$ & $27,3 \%$ \\
\hline Bajo manual & $5,9 \%$ & $15,3 \%$ & $27,5 \%$ & $45,8 \%$ & $29,8 \%$ \\
\hline Total & $100,0 \%$ & $100,0 \%$ & 100,0 & & \\
\hline
\end{tabular}

Fuente: elaborado en base a Jorrat (2001), p. 201. 